\title{
Uma Contribuição na Determinação das Variáveis da Corrente Contínua Pulsada para o Arame Tubular E71T-1M
}

\author{
Víctor Vergara Díaz ${ }^{1}$, Francisco Fernández Acevedo ${ }^{1}$, Tiago Vieira da Cunha² \\ ${ }^{1}$ Universidad de Antofagasta, Campus Coloso, Antofagasta, II Región, Chile. \\ ${ }^{2}$ Universidade Federal de Santa Catarina - UFSC, Campus Joinville, Joinville, SC, Brasil.
}

Recebido: 21 Mar.. 2018

Aceito: 09 Set., 2018

E-mail: victor.vergara@uantof.cl (VVD)
Resumo: Este trabalho tem por objetivo principal implementar o processo de soldagem FCAW com corrente contínua pulsada. Para tanto, foi analisada a influência da Distância de Bico de Contato-Peça (DBCP) em dois níveis (19 e $24 \mathrm{~mm}$ ) e o tamanho do diâmetro da gota (Dg) de acordo com o seguinte critério: Diâmetro da gota igual ao diâmetro do arame-eletrodo (Dg=De), diâmetro da gota menor que o diâmetro do arame-eletrodo ( $\mathrm{Dg}<\mathrm{De}$ ) e, finalmente, diâmetro da gota maior que o diâmetro do arame-eletrodo (Dg>De), mantendo a taxa de deposição fixa. A análise dos resultados obtidos revelou que a metodologia experimental empregada é efetiva na determinação das variáveis da corrente contínua pulsada para o arame tubular rutílico E71T-1M. De posse desta, foi possível produzir cordões de solda de bom aspecto superficial e morfológico, além de se obter boa estabilidade do arco (livre de respingos) quando utilizada uma Distância de Bico de Contato-Peça (DBCP) de $24 \mathrm{~mm}$ e diâmetro da gota (Dg) menor que o diâmetro do arame-eletrodo (De). Não obstante, quando empregada DBCP de $24 \mathrm{~mm}$, foi observada uma significativa variação na energia de soldagem quando empregado diferentes diâmetros de gota, sendo obtidos os valores de $9,0 \mathrm{~kJ} / \mathrm{cm}$ para Dg>De e $12,6 \mathrm{~kJ} / \mathrm{cm}$ para $\mathrm{Dg}<\mathrm{De}$. Isto representa um aumento de cerca de $40 \%$ quando são empregados diâmetros de gota menores que diâmetro do eletrodo (Dg<De).

Palavras-chave: FCAW-P; Arame tubular; Corrente pulsada.

\section{A Contribution in the Determination of the Pulsed Current Variables for the E71T-1M Tubular Wire}

\begin{abstract}
This work has as main objective to implement the FCAW welding process with pulsed current. For this purpose, the influence of nozzle distance (DBCP) on two levels (19 and $24 \mathrm{~mm}$ ) and droplet diameter (Dg) size were analyzed according to the following criteria: Droplet diameter equal to the diameter of the electrode wire $(\mathrm{Dg}=\mathrm{De})$, droplet diameter less than the diameter of the electrode wire $(\mathrm{Dg}<\mathrm{De})$ and, finally, droplet diameter larger than the diameter of the electrode wire ( $\mathrm{Dg}>\mathrm{De})$, without changind the deposition rate. The analysis of the results showed that the experimental methodology used is effective to determine the pulsed current variables for the E71T-1M rutile tubular wire. Thus, it was possible to produce weld beads with good surface and morphological appearance, as well as good arc stability (without weld spatter) when using a $24 \mathrm{~mm}$ of distance from the nozzle to the workpiece (DBCP) and smaller droplet diameter (Dg) than the diameter of the electrode-wire (De). Moreover, when using DBCP of $24 \mathrm{~mm}$, a significant variation in the welding energy was observed when different droplet diameters were used. About that, with $D g>$ De was obtained 9,0 kJ/cm and for $\mathrm{Dg}<\mathrm{De} 12.6 \mathrm{~kJ} / \mathrm{cm}$. This represents an increase of about $40 \%$ when droplet diameters smaller than the electrode diameter $(\mathrm{Dg}<\mathrm{De})$ are used.
\end{abstract}

Key-words: FCAW-P; Flux cored wire; Pulsed current.

\section{Introdução}

Devido as suas características, especialmente aquelas associadas à alimentação mecanizada do arame, o que favorece a automatização do processo [1,2] e a obtenção de maiores índices de produtividade, processos de soldagem como o GMAW e FCAW naturalmente despontam como os principais processos de soldagem a arco sendo utilizados industrialmente. Em vista disso, estes processos tem sido alvo de contínuo desenvolvimento em centros de pesquisas, sobretudo, com o intuito de aumentar sua capacidade produtiva ou o de melhorar suas características operacionais, tornando-os mais adequados em certas aplicações. Como resultado, ao longo das últimas décadas diversas técnicas de soldagem, ou variantes destes processos, surgiram, dentre elas a soldagem pulsada.
Este é um artigo publicado em acesso aberto (Open Access) sob a licença Creative Commons Attribution Non-Commercial, que permite uso, distribuição e reprodução em qualquer meio, sem restrições des de que sem fins comerciais e que 0 trabalho original seja corretamente citado. 
Desde o seu surgimento em 1962, a soldagem GMAW Pulsada tem sido amplamente utilizada tanto na soldagem mecanizada quanto na robotizada [3]. O processo GMAW Pulsado foi concebido com o objetivo de se reunir, em um só processo, as características desejáveis dos modos de transferência naturais por curto circuito e goticular. Deste modo, obtém-se, assim, uma transferência estável, que proporciona baixos níveis de respingos e bom acabamento do cordão, associada a um baixo valor de corrente média de soldagem. Isto é conseguido com a alternância periódica do valor da corrente de soldagem entre dois níveis bem definidos (corrente de pulso - Ip e corrente de base - lb), com o intuito de controlar a transferência metálica. O valor da corrente de base empregado é muito baixo para que ocorra a transferência metálica [4]. Deste modo, enquanto esta corrente atua, ocorre a formação de uma gota de metal líquido na extremidade do arame ao mesmo tempo em que se mantém o arco aberto em menores valores de corrente, contribuindo, assim, para uma menor corrente média de soldagem. Já o valor da corrente de pulso, necessariamente maior que a corrente de transição globular-goticular, tem como função atuar no destacamento da gota metálica formada previamente na ponta do arame, fazendo uso, principalmente, da força eletromagnética [5]. Daí a necessidade do emprego de elevados valores para a corrente de pulso.

Um aspecto importante da soldagem pulsada diz respeito ao expressivo aumento no número de variáveis de soldagem a serem empregadas em comparação ao processo GMAW convencional. Não obstante, estes parâmetros, a saber; corrente de pulso (Ip), corrente de base (Ib), tempo de pulso (tp), tempo de base (tb) e velocidade de alimentação de arame (Va) devem, necessariamente, ser selecionados de forma concatenada de modo a assegurar o adequado comportamento operacional do processo e, assim, se obter a estabilidade do arco e a qualidade dos cordões de solda típicos desta técnica.

Acredita-se que esta seja a principal razão pelo qual a soldagem GMAW pulsada enfrenta resistência no âmbito industrial, especialmente no Chile. Apesar de atualmente existirem disponíveis no mercado uma variedade bastante grande de equipamentos de soldagem com programas sinérgicos, a falta de conhecimento dos profissionais que atuam na indústria ainda se constitui na grande barreira para que esta técnica de soldagem tenha uma aplicação mais abrangente. Naturalmente, por outro lado, no âmbito acadêmico isto não acontece. Mesmo para aqueles que estão iniciando na pesquisa acadêmica em soldagem, existem disponíveis na literatura especializada trabalhos como o de Palani e Murugan [4] que abordam, de forma sistematizada, a definição de tais variáveis para uma determinada condição de soldagem. Sendo assim, também é possível encontrar na literatura uma vasta quantidade de trabalhos que abordam o emprego da corrente pulsada na soldagem GMAW. Contudo, em se tratando da soldagem FCAW pulsada o cenário é um pouco diferente. Além de não existir a mesma quantidade de trabalhos disponíveis, na grande maioria dos casos estes abordam a pulsação da corrente no processo FCAW com base na comparação com o GMAW ou com o processo FCAW convencional. Neste contexto, têm-se o trabalho de Krishnan et al. [6] que realizaram um estudo quanto ao emprego da corrente pulsada em arames tubulares. De um modo geral, o estudo mostrou que a soldagem FCAW com corrente pulsada é capaz de aumentar a taxa de deposição em $42 \%$ e velocidade de soldagem em $20 \%$, além de conferir melhores propriedades mecânicas nos cordões de solda resultantes, quando comparado com a soldagem com arames maciços. Já French e Bosworth [7] conduziram um estudo com arames tubulares a fim de comparar as transferências obtidas no modo convencional e com corrente pulsada. De acordo com seus resultados, quando utilizada a corrente pulsada foi possível identificar uma maior faixa operacional de correntes de soldagem, particularmente, em baixos valores de corrente média, enquanto que melhor acabamento e molhabilidade nos cordões de solda obtidos tanto nas posições de soldagem vertical como horizontal. Ainda em se tratando dos efeitos da corrente pulsada, Di et al. [8] estudaram as propriedades mecânicas da solda e a morfologia das dendritas. Estes autores concluíram que o uso da corrente pulsada promove uma vibração efetiva na poça de fusão, acarretando, assim, em mudanças no estado de solidificação do metal de solda. Deste modo, as condições de segregação são melhoradas e, portanto, produzindo pequenas dendritas, que são benéficas para com a ductilidade da junta soldada.

Conforme discutido, embora existam na literatura trabalhos que tratam da pulsação da corrente no processo FCAW, destes, poucos são aqueles que estão dirigidos ao comportamento da transferência metálica e sua influência na estabilidade do processo e comportamento dos cordões de solda resultantes. Deste modo, o presente trabalho tem como objetivo determinar, para um dado arame tubular, o conjunto de variáveis da corrente pulsada de acordo a diretrizes definidas na literatura para, assim, analisar a influência do tamanho da gota sobre os aspectos morfológicos dos cordões de solda e da estabilidade do processo. 


\section{Materiais e Métodos}

Para a realização do presente trabalho foi concebida uma bancada de ensaios constituída por uma fonte de soldagem multiprocesso modelo DIGIPLUS A7. A tocha utilizada é refrigerada à agua com capacidade de corrente de 450 A. A mesma foi instalada num sistema de deslocamento com quatro graus de liberdade (Figura 1). O monitoramento da corrente de soldagem e da tensão de arco foram realizados com o sistema de aquisição próprio da fonte de soldagem. Os cordões de solda foram executados na posição plana com ângulo de ataque da tocha de $90^{\circ}$, utilizando como material base corpos de prova, com dimensões de 5x50×216 mm, do aço SAE 1015 . O material de adição empregado foi o AWS E71T-1M de 1,6 mm de diâmetro e como gás de proteção a mistura Ar+20\% $\mathrm{CO}_{2}$, $\mathrm{Conforme}$ recomendação do fabricante do arame. Foram analisadas a Distância de Bico de Contato-Peça (DBCP de 19 e 24 mm) e o tamanho da gota (Diâmetro Dg) em relação ao diâmetro do arame-eletrodo (De) da seguinte forma: Dg=De, Dg>De e Dg<De. As variáveis de resposta foram a estabilidade do processo, morfologia e aspecto superficial dos cordões de solda e o grau de respingos. Este último avaliado por intermédio de inspeção visual.

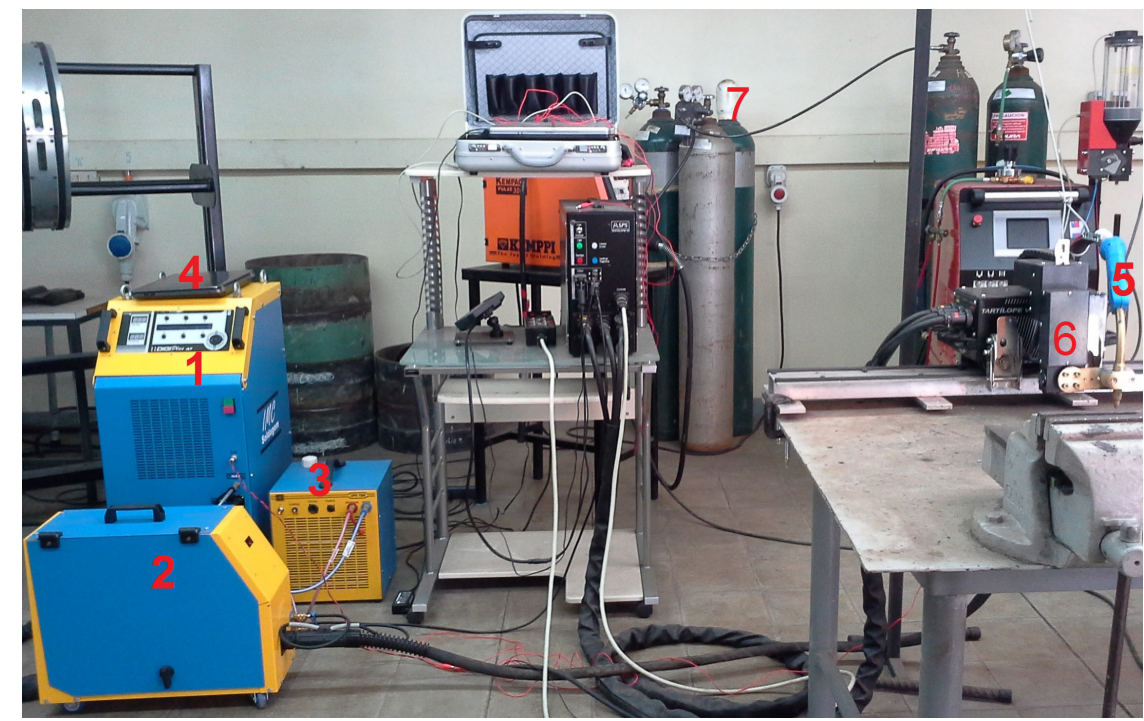

Figura 1. Bancada de ensaios utilizada no trabalho. 1-Fonte de soldagem, 2-Alimentador de arame, 3-Sistema de refrigeração da tocha, 4-Sistema de aquisição de dados, 5-Tocha, 6-Sistema de deslocamento da tocha e 7-Gases de proteção.

\subsection{Determinação da corrente de transição}

Seguindo a metodologia empregada na literatura [1], foi necessário primeiramente realizar ensaios preliminares em corrente contínua constante (Ensaio $N^{\circ} 1$ ) com o intuito de identificar a corrente de soldagem necessária para que ocorra a transferência goticular. Na Tabela 1 são apresentados os parâmetros utilizados na realização destes ensaios.

Tabela 1. Parâmetros de soldagem empregados nos ensaios com transferência goticular.

\begin{tabular}{cc} 
Parâmetro & Valor \\
Gás & Ar + 20\% CO2 \\
Vazão de gás & $16 \mathrm{~L} / \mathrm{min}$ \\
Distância de Bico de Contato-Peça (DBCP) & $24 \mathrm{~mm}$ \\
Velocidade de arame - Va & $4,5 \mathrm{~m} / \mathrm{min}$ \\
Velocidade de soldagem - Vs & $20 \mathrm{~cm} / \mathrm{min}$ \\
Arame & $\mathrm{E} 71 \mathrm{~T}-1 \mathrm{M}$ \\
Bitola do arame & $1,6 \mathrm{~mm}$ \\
Tensão de referência - Ur & $22 \mathrm{~V}$ \\
Posição de soldagem & Plana \\
\hline
\end{tabular}


Na Figura 2 estão presentes os oscilogramas de tensão de arco e de corrente de soldagem onde é possível identificar uma corrente média de cerca de 250 A. Uma vez identificada a corrente de transição, o passo seguinte foi utilizar a Equação 1 para determinar a constante de consumo $\mathrm{K}=0,018 \mathrm{~m} \cdot \mathrm{min}^{-1}$. $\mathrm{A}^{-1}$. A corrente de pulso empregada deve ser superior á corrente de transição, portanto, nos ensaios com corrente pulsada adotou-se uma corrente de pulso (Ip) igual a $300 \mathrm{~A}$ e tempo de pulso (tp) igual a $5 \mathrm{~ms}$, dados que permitem, mediante a Equação 2, determinar o destacamento de uma gota por pulso $\mathrm{Ip}^{2} . \mathrm{tp}=420,5 \mathrm{~A}^{2}$.s.

$$
\begin{aligned}
& V a=K . I m \\
& I p^{2} . t p=D
\end{aligned}
$$

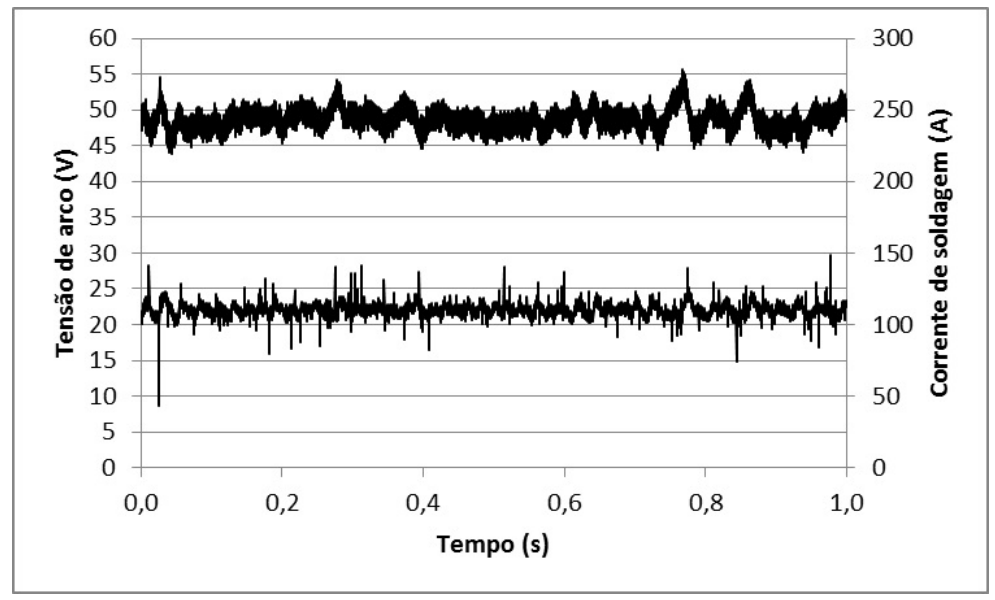

Figura 2. Oscilogramas de tensão e corrente do ensaio $\mathrm{N}^{\circ} 1 . \mathrm{Ur}=22 \mathrm{~V}, \mathrm{Va}=4,5 \mathrm{~m} / \mathrm{min}$.

\subsection{Influência do tamanho da gota}

Nos ensaios seguintes foi analisada a influência do tamanho da gota (Dg) sobre a estabilidade do processo para variações na Distância de Bico de Contato-Peça (DBCP) em dois níveis 19 e 24 mm. Experimentos realizados por Nogueira et al. [1] mostram que a seleção do diâmetro da gota com valor de $20 \%$ maior que o diâmetro do arame-eletrodo satisfaz a maioria das aplicações, deste modo, foi utilizado um Dg de 1,92 mm. Seguindo com este critério, para valores de tamanho da gota menores que o diâmetro do arame-eletrodo foi utilizado 1,28 mm. O período T da corrente contínua pulsada é o tempo necessário para obter um determinado diâmetro da gota (Dg) quando é utilizada uma determinada velocidade de arame (Va) e diâmetro de eletrodo (De). A Equação 3 permitiu realizar o cálculo do período de tempo T em que o volume de arame que avança durante um determinado tempo se transforma em uma gota de diâmetro Dg.

$$
T=t p+t b=\frac{40 \cdot D_{g}{ }^{3}}{V a \cdot D_{e}^{2}}
$$

Em seguida foi determinado o tempo de base tb sabendo que $\mathrm{T}=\mathrm{tb}+\mathrm{tp}$. Finalmente com a Equação 4 , que define a corrente média de soldagem, foi determinada a corrente de base (Ib).

$$
\operatorname{Im}=\frac{\operatorname{Ip} \cdot t p+I b \cdot t b}{T}
$$

Na Tabela 2 constam os parâmetros da corrente contínua pulsada utilizados para os três possíveis critérios do diâmetro da gota em relação ao diâmetro do arame-eletrodo. Estes parâmetros calculados foram programados na fonte de soldagem e os cordões de solda foram realizados de forma mecanizada e monitorados pelo sistema de 
aquisição de dados. Na Tabela 2 também é possível observar que para um diâmetro da gota menor que o diâmetro do arame-eletrodo foram alcançadas as maiores frequências de pulsação, possibilitando, deste modo, um arco mais estável. Os demais parâmetros empregados na soldagem pulsada são os mesmos contidos na Tabela 1, exceto a velocidade de soldagem (Vs) que neste caso foi de $20 \mathrm{~cm} / \mathrm{min}$.

Tabela 2. Parâmetros da corrente contínua pulsada em função do diâmetro da gota.

\begin{tabular}{|c|c|c|c|c|c|c|c|c|}
\hline Ensaio No & Diâmetro da gota - Dg & $\begin{array}{l}\text { DBCP } \\
(\mathrm{mm})\end{array}$ & $\begin{array}{l}\text { Ip } \\
\text { (A) }\end{array}$ & $\underset{(m s)}{t p}$ & $\begin{array}{l}\text { Ib } \\
\text { (A) }\end{array}$ & $\begin{array}{c}\text { tb } \\
\text { (ms) }\end{array}$ & $\begin{array}{c}f \\
(H z)\end{array}$ & $\begin{array}{l}\text { Im } \\
\text { (A) }\end{array}$ \\
\hline 2 & $\mathrm{Dg}=\mathrm{De}$ & \multirow{4}{*}{20} & \multirow{6}{*}{300} & \multirow{6}{*}{5} & 125,85 & 16,3 & 46,9 & 167 \\
\hline 3 & $\mathrm{Dg}>\mathrm{De}$ & & & & 145,7 & 31,8 & 27,1 & 167 \\
\hline 4 & $\mathrm{Dg}<\mathrm{De}$ & & & & 54,1 & 5,9 & 91,6 & 167 \\
\hline 5 & $\mathrm{Dg}<\mathrm{De}$ & & & & 54,1 & 5,9 & 91,6 & 167 \\
\hline 6 & $\mathrm{Dg}>\mathrm{De}$ & \multirow[t]{2}{*}{15} & & & 145,7 & 31,8 & 27,1 & 167 \\
\hline 7 & $\mathrm{Dg}=\mathrm{De}$ & & & & 125,85 & 16,3 & 46,9 & 167 \\
\hline
\end{tabular}

De: Diâmetro do arame-eletrodo; DBCP: Distância de Bico de Contato-Peça; Ip: Corrente de pulso; tp: Tempo de pulso; f: Frequência; Im: Corrente média.

Conforme as diretrizes disponíveis na literatura acerca da metodologia empregada para determinar as variáveis da corrente pulsada [1] e à experiência adquirida no desenvolvimento do presente trabalho, foi possível conceber uma representação esquemática do planejamento experimental (Figura 3). Este esquema mostra o fluxograma (1) com as equações e critérios para determinar as variáveis da corrente pulsada. As equações e critérios deste diagrama foram programados numa planilha Excel (2) e os valores das variáveis obtidos inseridos na fonte de soldagem (3). Durante a execução da soldagem (4) as variáveis foram monitoradas mediante o sistema de aquisição de dados para seu controle (5).

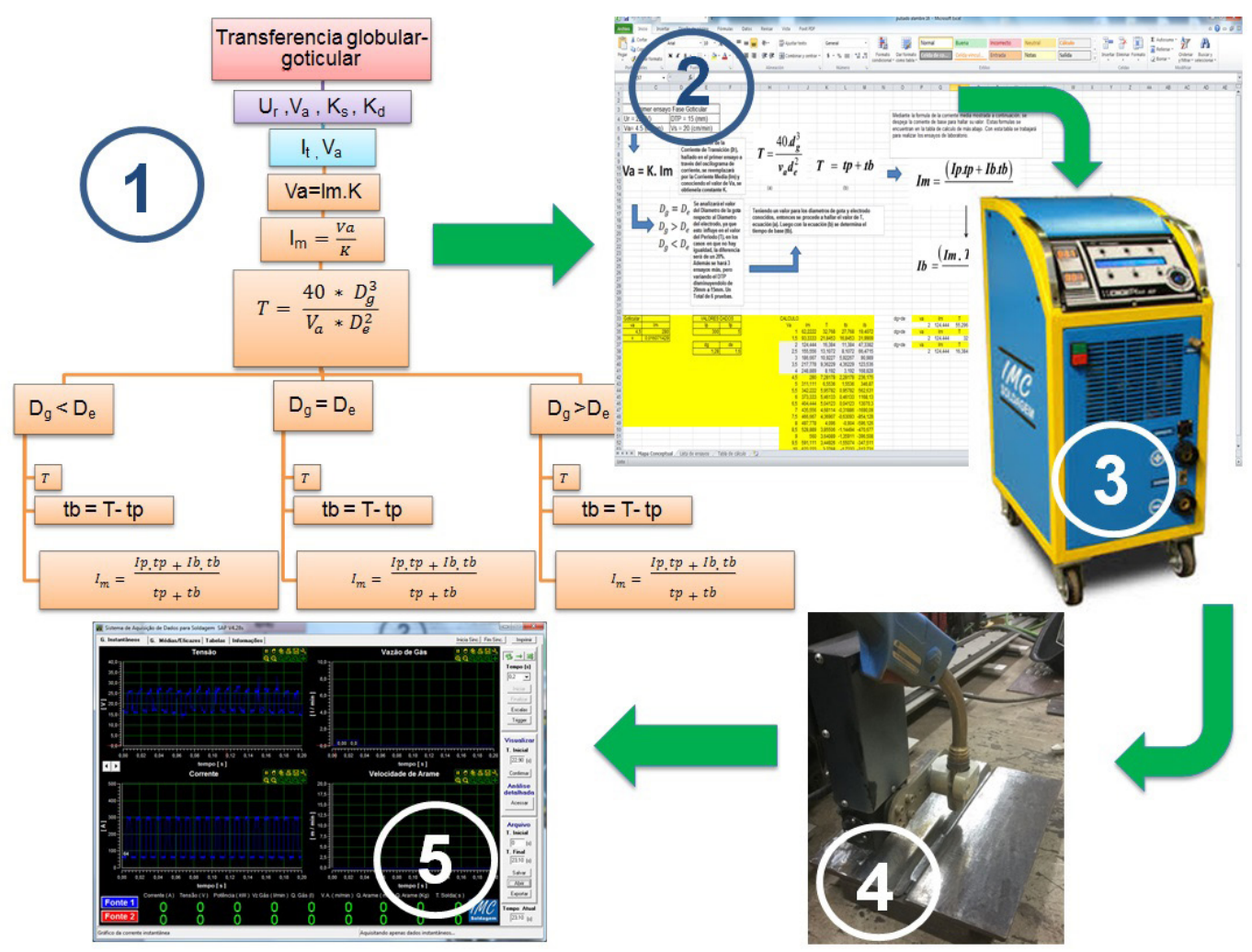

Figura 3. Representação esquemática do planejamento experimental adotado na determinação das variáveis da corrente pulsada. 1-Fluxograma, 2-Planilha Excel, 3-Fonte de soldagem, 4-Execução da soldagem, 5-Monitoramento mediante o sistema de aquisição de dados para seu controle. 


\section{Resultados e Discussão}

\subsection{Energia de soldagem}

A Figura 4a apresenta a energia de soldagem para a corrente contínua pulsada onde é possível observar uma sensível diferença de energia quando foi variada a DBCP. Conforme esperado, os maiores valores de energia foram obtidos para DBCP de $24 \mathrm{~mm}$, devido ao maior comprimento do arco nesta configuração. Relativo a isto, na Figura 4b são apresentados os valores médios de tensão de arco para as variações na Distância de Bico de Contato-Peça (DBCP), mantendo constante a velocidade de alimentação de arame (Va).

Ainda na Figura 4a, observa-se uma significativa variação na energia de soldagem quando os cordões de solda são realizados com diferentes diâmetros da gota (Dg). É importante destacar que o tamanho da gota está intimamente relacionado à frequência de pulsação da corrente (Tabela 2). Neste trabalho, os maiores valores de energia de soldagem foram encontrados para o caso $\mathrm{Dg}<\mathrm{De}$, ou seja, quando a transferência metálica ocorre em maiores frequências de destacamento de gotas. Resultados semelhantes foram encontrados por Santos et al. [9] revelando que quando existe um aumento na frequência de pulsação, se tem um aumento na tensão do arco e como consequência um aumento na energia do mesmo.
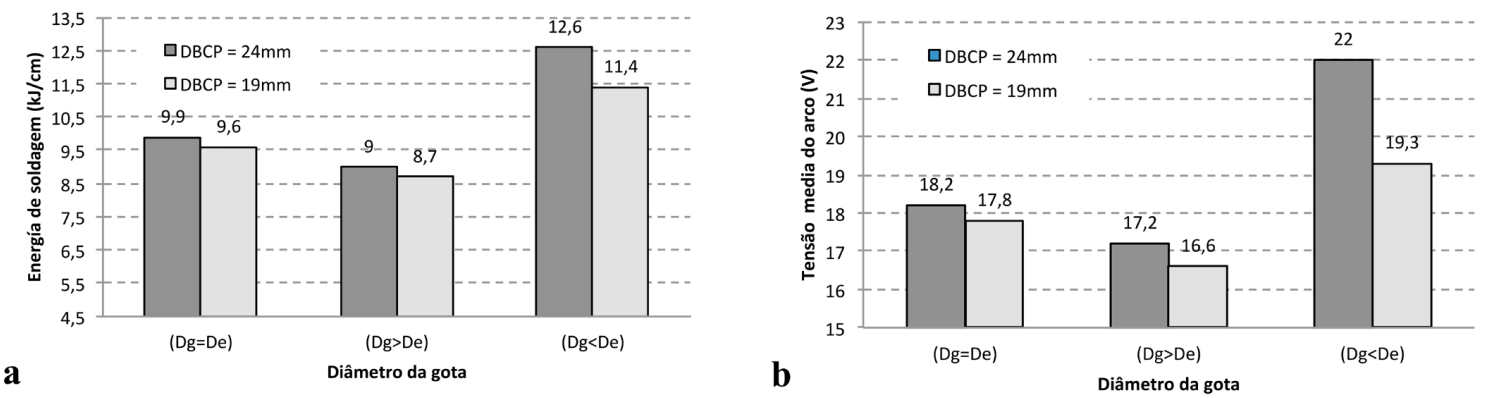

Figura 4. (a) Energia de soldagem e (b) Tensão média de arco em função da DBCP e o diâmetro da gota (Dg). DBCP: Distância de Bico de Contato-Peça.

\subsection{Morfologia e aspecto superficial dos cordões de solda}

A Figura 5 mostra o aspecto superficial dos cordões de solda realizados de acordo com o planejamento dos ensaios contido na Tabela 2. Conforme pode ser visto, os cordões de solda dos ensaios 2, 3, 6 e 7 apresentam um índice considerável de respingos em se tratando de soldagem pulsada, enquanto que o cordão do ensaio 5 um menor grau de respingos. De fato, os melhores resultados foram obtidos para um diâmetro de gota menor que o diâmetro do arame-eletrodo e, sobretudo, com uma maior altura de arco (Ensaio 4). Neste ensaio o cordão de solda apresentou um bom aspecto superficial e, principalmente, uma quantidade de respingos muito baixa.

Na Figura 6 é apresentada a seção transversal dos cordões de solda obtidos nos ensaio 2 a 7. Nesta, ao se realizar uma análise qualitativa é possível perceber, para todos os ensaios, um baixo perfil de penetração e um bom índice de convexidade. Apesar das maiores potências de arco, obtidas com DBCP igual a 24 mm devido a maior altura de arco, verifica-se que os cordões de solda realizados nesta condição apresentam índices de penetração muito semelhantes àqueles cordões realizados com DBCP igual a $19 \mathrm{~mm}$.

Já na Figura 7 são apresentados os valores de largura, reforço e penetração dos cordões de solda para os dois níveis de Distância de Bico de Contato-Peça (DBCP) empregados e para os três critérios do diâmetro da gota (Dg) estabelecidos no trabalho. Os resultados indicam que a DBCP apresenta influência sobre a largura (L) e o reforço (R) dos cordões somente para o caso em que $\mathrm{Dg}>\mathrm{De}$, acarretando, nesta condição, num aumento de cerca de $10 \%$ nos valores obtidos para estas dimensões. Por outro lado, para Dg < De a influência de DBCP é significativa no valor da penetração. Acredita-se que isto se deve, possivelmente, à maior frequência de pulsação da corrente de soldagem obtida para $\mathrm{Dg}<\mathrm{De}$. Deste modo, apesar do valor de corrente média permanecer inalterado, supõe-se que uma maior pressão média do arco é obtida. Isto, associado à maior energia do arco obtida com o DBCP de 24 mm (como resultado da maior tensão de arco resultante) conduz, portanto, ao aumento de penetração observado. 


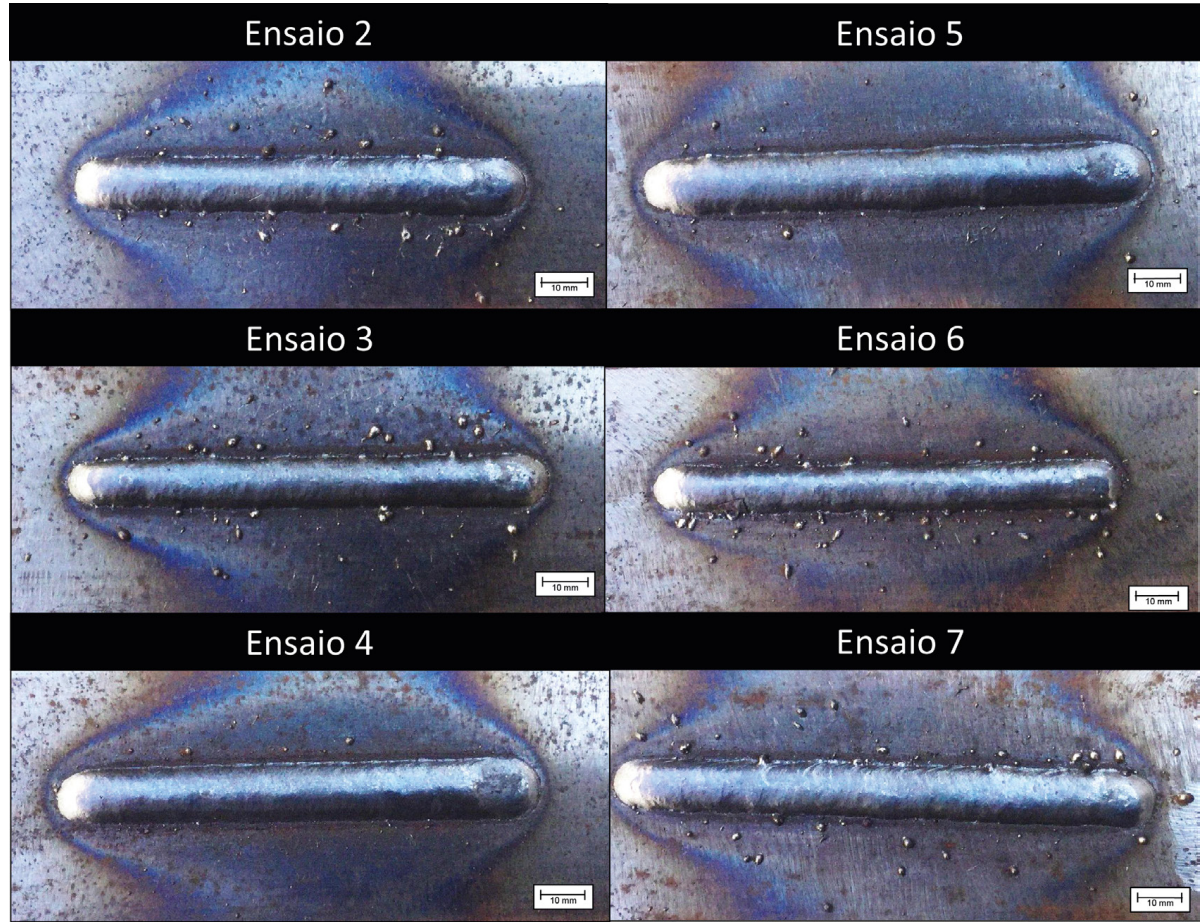

Figura 5. Aspecto superficial dos cordões de solda.
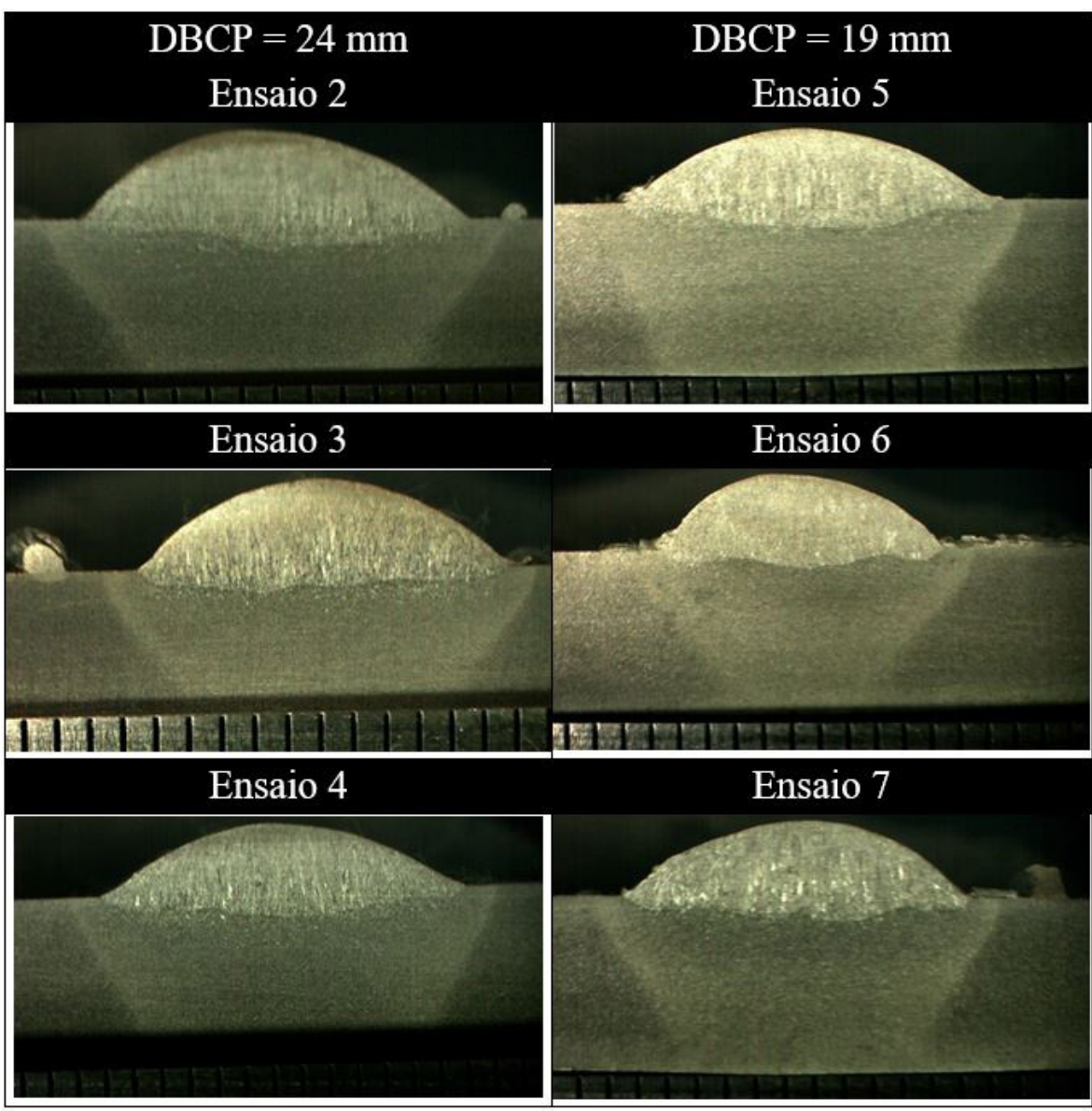

Figura 6. Macrografias correspondentes aos ensaios da Tabela 2. DBCP: Distância de Bico de Contato-Peça. 

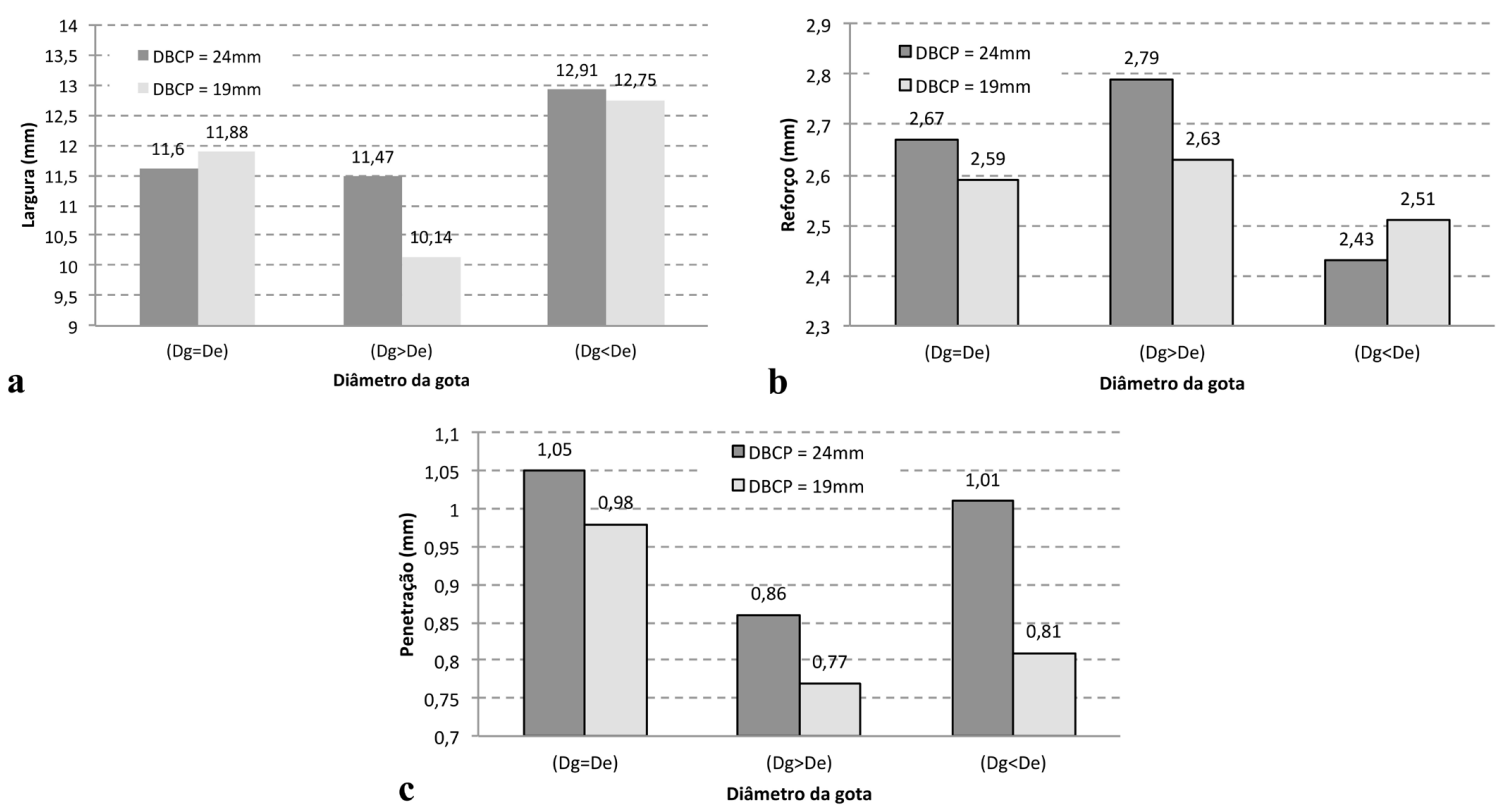

Figura 7. (a) Largura, (b) Reforço e (c) Penetração dos cordões de solda em função da DBCP e do diâmetro de gota (Dg). DBCP: Distância de Bico de Contato-Peça.

Resultados semelhantes foram encontrados por Mohamat et al. [10] onde evidenciam a influência que a tensão do arco possui no perfil de penetração do cordão de solda.

\subsection{Análise da estabilidade do processo.}

Na Figura 8 são apresentados os oscilogramas de corrente de soldagem e de tensão de arco obtidos nos ensaios 2 a 7.

Conforme pode ser visto, para os casos em que o diâmetro da gota é igual ou superior ao diâmetro do arame-eletrodo (ensaios 2, 3, 7 e 6), têm-se um processo com muitas instabilidades. Já no oscilograma do ensaio 4, caso em que o diâmetro da gota é menor que o diâmetro do arame-eletrodo, se observa uma clara estabilidade do processo, acompanhada do aumento na frequência de pulsação da corrente. No entanto, o mesmo não acontece para o ensaio 5, apesar deste também ser realizado com um diâmetro de gota menor. Analisando esses oscilogramas, verifica-se que as instabilidades observadas nos ensaios 2, 3, 7 e 6 se deve, possivelmente, ao tempo de pulso empregado ser insuficiente para proporcionar a expulsão das gotas (de volume considerável) a partir da ponta do arame. Deste modo, conforme pode ser verificada nesses oscilogramas, a transferência metálica acaba ocorrendo durante o período de base seguinte ao pulso, instabilizando, portanto, a execução do processo. Neste sentido, na medida em que o volume das gotas diminui (ensaios 2 e 7), um menor tempo de pulso é requerido para que ocorra a transferência metálica. Por fim, quando Dg se torna menor que De, o tempo de pulso empregado (5 ms) se mostra suficiente para destacar estas gotas de menor volume e, assim, se alcançar a estabilidade do processo almejada (ensaio 4). Isto posto, era de se observar a mesma estabilidade do processo nos oscilogramas do ensaio 5 , entretanto, neste, as instabilidade presentes possivelmente são decorrente da altura de arco insuficiente. Fato evidenciado pelos curtos-circuitos observados no final do período de base/início do período de pulso. Assim, antes da ocorrência da transferência metálica, pela ação da corrente de pulso, a gota toca a superfície da poça de fusão instabilizando, portanto, o processo.

A fim de melhor avaliar a estabilidade do processo em questão, foi utilizada a ferramenta denominada de ciclograma. Com esta ferramenta se torna mais fácil analisar a repetitividade do processo e, assim, inferir sobre o seu comportamento. A Figura 9 apresenta os ciclogramas de tensão-corrente obtidos nos ensaios 2,3 e 4 . Conforme pode ser visto nas Figuras 9a e 9b, existem linhas dispersas evidenciando, assim, uma falta de repetitividade do processo e, portanto, uma condição instável de operação do processo. Por outro lado, têm-se o oscilograma da Figura 9c, no qual as linhas encontram-se concentradas numa única região do gráfico, corroborando, assim, a análise anteriormente realizada via oscilograma. 


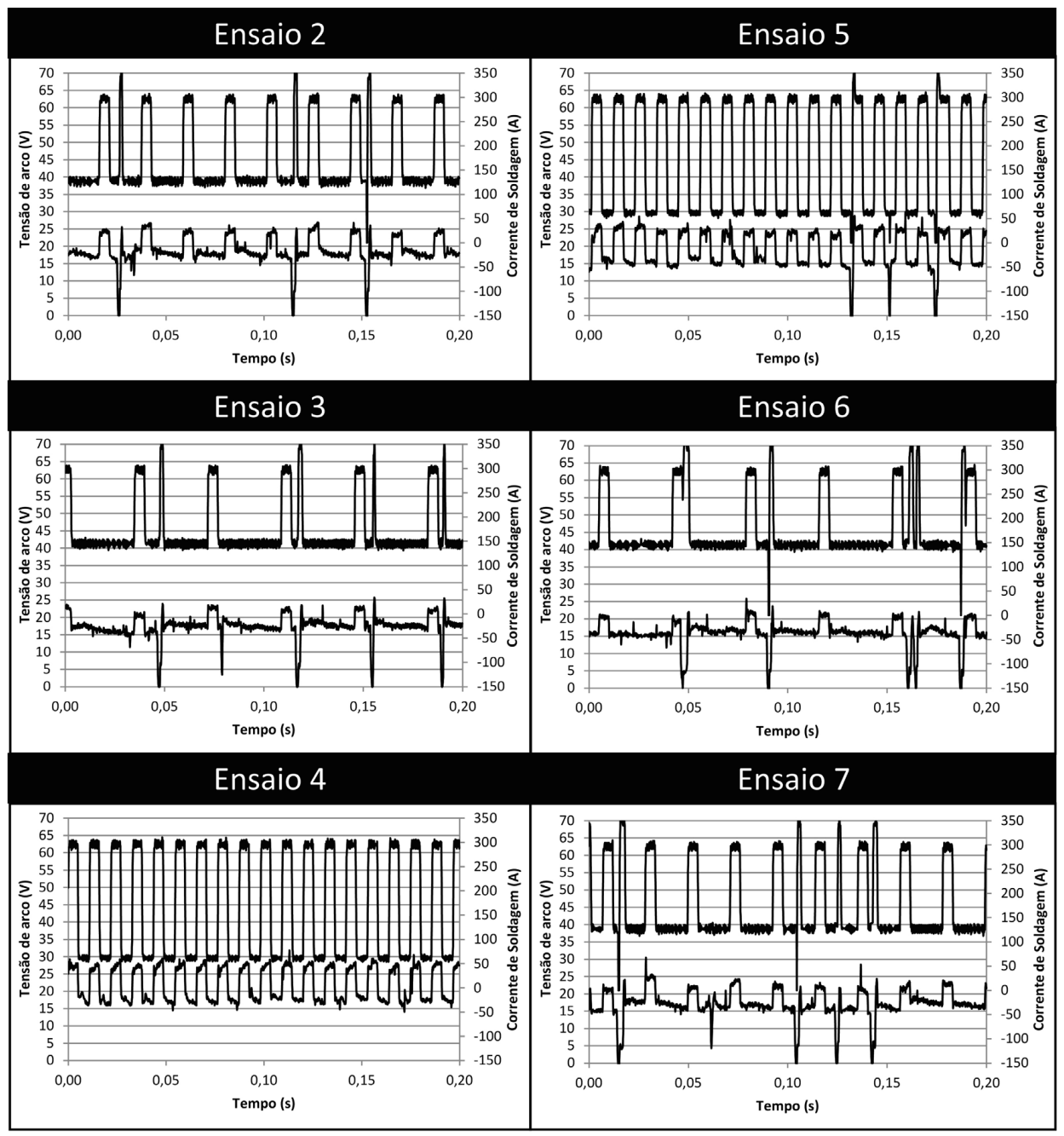

Figura 8. Oscilogramas de Corrente de soldagem e Tensão de arco obtidos nos ensaios 2 a 7.
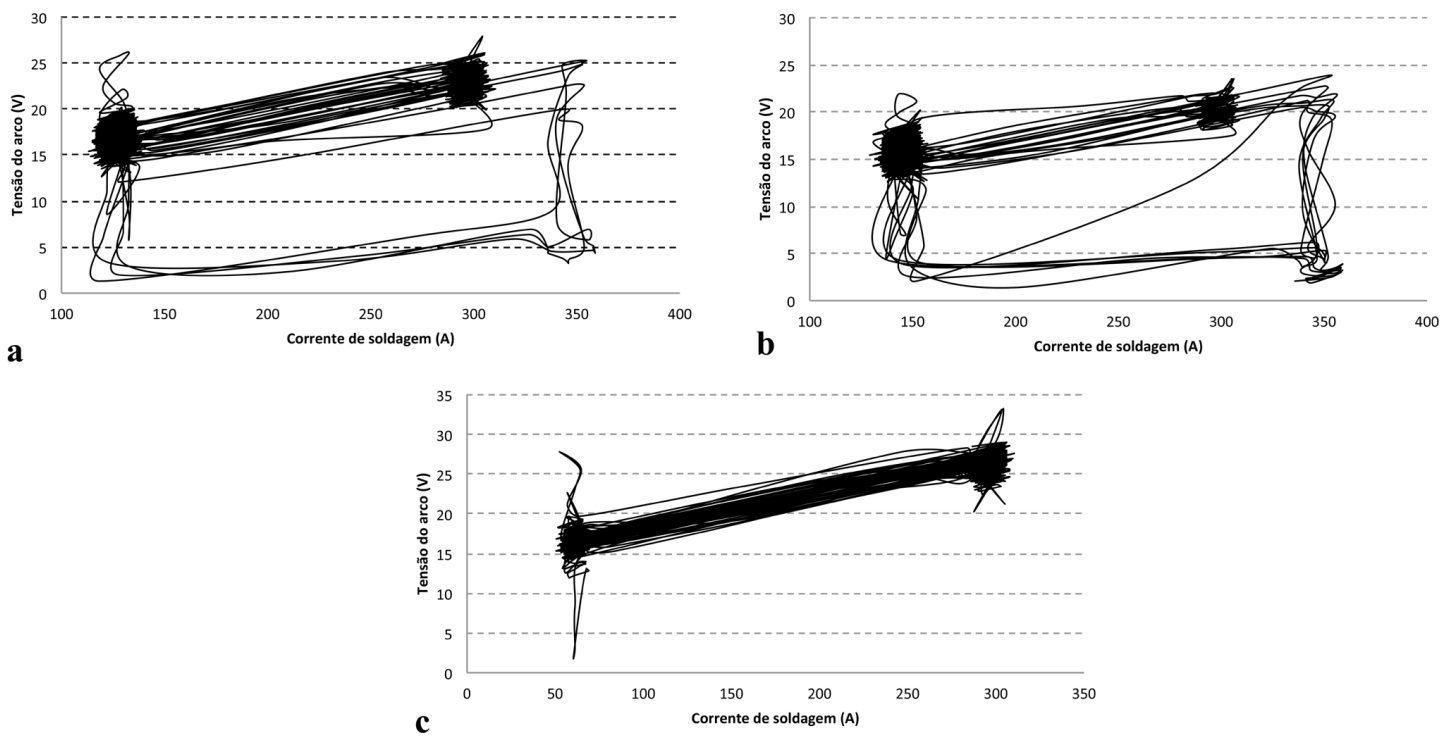

Figura 9. Ciclogramas Tensão-corrente obtidos nos ensaios (a) 2, (b) 3 e (c) 4. 


\section{Conclusões}

Com base nos resultados obtidos, podemos concluir que:

- A determinação experimental das variáveis relativas à soldagem com corrente pulsada para o arame tubular AWS E71T-1M de 1,6 mm de diâmetro foi possível mediante a metodologia empregada;

- Com as variáveis empregadas foi possível obter uma excelente estabilidade do processo, conduzindo a obtenção de soldagem isentas de respingos quando empregada uma Distância de Bico de Contato-Peça (DBCP) de $24 \mathrm{~mm}$;

- Ao empregar uma Distância de Bico de Contato-Peça de 24 mm foi observada uma significativa variação na energia de soldagem para diferentes diâmetros de gota, obtendo-se valores de 9,0 kJ/cm para Dg>De e de $12,6 \mathrm{~kJ} / \mathrm{cm}$ para $\mathrm{Dg}<\mathrm{De}$. Isto representa um aumento da energia de soldagem de cerca de $40 \%$ quando empregados diâmetros de gota menores que o diâmetro do eletrodo ( $\mathrm{Dg}<\mathrm{De}$ ). Esta variação na energia de soldagem afetou, portanto, a geometria do cordão de solda, acarretando num aumento de largura e penetração do cordão de cerca de $12 \%$ e 17\%, respectivamente, além de uma diminuição do reforço de cerca de $15 \%$.

\section{Agradecimentos}

Os autores agradecem à empresa Minetec Ltda. - Chile e à empresa IMC Soldagem - Brasil pelo auxílio na elaboração deste trabalho.

\section{Referências}

[1] Nogueira RMU, Bohórquez CEN, Zanella IG. Comparação da estabilidade do arco e da variabilidade da geometria de soldas obtidas pelos processos MIG/MAG e arame tubular. Soldagem e Inspeção. 2015;20(2):191-204. http://dx.doi.org/10.1590/01049224/SI2002.07.

[2] Addamani R, Ravindra HV, Ugrasen G, Kumar NP, Darshan CS. Estimation and comparison of welding performances using MRA and GMDH in P-GMAW for ASTM 106 material. Materials Today: Proceedings. 2018;5(1):2985-2993. http://dx.doi.org/10.1016/j. matpr.2018.01.097

[3] Kim S, Eagar TW. Metal transfer in pulsed current gas metal arc welding. Welding Journal (Suppl.):279s-287s. 1993.

[4] Palani PK, Murugan N. Selection of parameters of pulsed current gas metal arc welding. Journal of Materials Processing Technology. 2006;172(1):1-10. http://dx.doi.org/10.1016/j. jmatprotec.2005.07.013.

[5] Scotti A, Ponomarev V. Soldagem MIG/MAG Melhor entendimento Melhor desempenho. São Paulo: ArtLiber. 2008.
[6] Krishnan S, Kulkarni DV, De A. Pulsed current gas metal arc welding of P91 steels using metal cored wires. Journal of Materials Processing Technology. 2016;229:826-833. http:// dx.doi.org/10.1016/j.jmatprotec.2015.11.007.

[7] French IE, Bosworth MR. A comparison of pulsed and conventional welding with basic flux cored and metal cored welding wires. Welding Journal. 1995;(Suppl.):197-205.

[8] Di X, Deng S, Wang B. Effect of pulse current on mechanical properties and dendritic morphology of modified medium manganese steel welds metal. Materials \& Design. 2015;66:169175. http://dx.doi.org/10.1016/j.matdes.2014.10.050.

[9] Santos EBF, Pistor R, Gerlich AP. High frequency pulsed gas metal arc welding (GMAW-P): the metal beam process. Manufacturing Letters. n. 11, p. 1-4, 2017. http://dx.doi.org/10.1016/j. mfglet.2017.01.001.

[10] Mohamat SA, Ibrahim IA, Amir A, Ghalib A. The Effect of Flux Core Arc Welding (FCAW) processes on different parameters. Procedia Engineering. 2012;41:1497-1501. http://dx.doi. org/10.1016/j.proeng.2012.07.341. 